\title{
Auditory event-related potentials are related to cognition at preschool age after very preterm birth
}

\author{
Holger Hövel' ${ }^{1}$ Eino Partanen ${ }^{2,3}$, Eva Tideman ${ }^{4}$, Karin Stjernqvist ${ }^{4}$, Lena Hellström-Westas ${ }^{5}$, Minna Huotilainen ${ }^{3,6}$ and \\ Vineta Fellman ${ }^{1,7}$
}

BACKGROUND: Auditory event-related potentials (AERP) are neurophysiological correlates of sound perception and cognitive processes. Our aim was to study in very preterm born children at preschool age if AERP correlate with cognitive outcome.

METHODS: Seventy children (mean \pm SD gestational age $27.4 \pm 1.9 w k$, birth weight $996 \pm 288 \mathrm{~g}$ ) were investigated at age 4.3-5.3 y with psychological testing (WPPSI-R, four subtests of NEPSY). Electroencephalogram was recorded while they listened to a repeated standard tone, randomly replaced by one of three deviants. Latencies and amplitudes for AERP components and mean amplitudes in successive 50-ms AERP time windows were measured.

RESULTS: Better cognitive test results and higher gestational age correlated with shorter P1 latencies and more positive mean amplitudes 150-500 ms after stimulus change onset. Neonatal brain damage was associated with a negative displacement of AERP curves. Neonatal morbidity had an impact on earlier time windows while gestational age and brain damage on both early and later time windows.

CONCLUSION: AERP measures were associated with cognitive outcome. Neonatal morbidity mainly affects early cortical auditory encoding, while immaturity and brain damage additionally influence higher cortical functions of auditory perception and distraction. Perinatal auditory environment might play a role in development of auditory processing.

$\mathbf{P}$ reterm children born at a gestational age less than $32 \mathrm{wk}$, even without neuromotor abnormalities, have as a group a lower mean intelligence quotient, an increased risk of neuropsychological deficits (such as attention, reading, learning, language, and memory impairment), neurosensory and visuospatial deficits, poor executive functions, behavioral problems, and low academic achievement $(1,2)$. The structural and functional correlates of such impairments in preterm children are incompletely understood, and early diagnostic tools are needed, but still lacking.
Auditory event-related potentials (AERP) are measures of cortical brain activity related to auditory stimuli. They are neurophysiological correlates of cortical sound discrimination and sound processing and may be used to describe auditory system developmental plasticity (3).

Obligatory AERP responses reflect sound detection and the transient encoding of the physical stimulus features (4). They are highly dependent upon the acoustic characteristics of the stimulus and the integrity of the primary auditory pathway $(2,5)$. At preschool age, they consist of a first positive peak around $100 \mathrm{~ms}(\mathrm{P} 1)$ and a second negative peak (N2) around $250 \mathrm{~ms}$ (2). Lower P1 and N2 amplitudes have been described in children with cognitive and/or behavioral problems $(6,7)$. Both in preterm and full term born children, lower P1 and N2 amplitudes have been associated with less favorable outcome, especially in language domains $(2,8)$.

Mismatch negativity (MMN) is an AERP component elicited by a change in auditory stimuli. It is a frontocentral distributed negativity peaking between 100 and $250 \mathrm{~ms}$ from the onset of a deviant stimulus, obtained by subtracting the average AERP to a frequent (standard) stimulus from that recorded after a rare (deviant) stimulus. The MMN, reflecting sound discrimination, is based on the automatic formation of neural memory traces for familiar auditory events and has been associated with preattentive cognitive processes in audition (9). While it is typically negative in adults, it may have a positive polarity in infants and young children (10). For salient sound changes, MMN is followed by the frontocentral P3a response, believed to represent an involuntary switch of attention (11).

In infants and children, pathological AERPs have been associated with a variety of neurocognitive impairments that have a high incidence after preterm birth $(6,12,13)$. The survival rate of extremely preterm infants $(<28$ gestational weeks $(\mathrm{GW}))$ continues to increase, which might be associated with an increased prevalence of neurocognitive abnormalities (14). In preterm infants with birth weights below 1,500 g, AERP development during the first year of life differed from healthy term infants (15). Smaller P1 and larger N2 amplitudes were found in

'Department of Pediatrics, Clinical Sciences, Lund and Skåne University Hospital, Lund University, Lund, Sweden; ${ }^{2}$ MINDLab - Center of Functionally Integrative Neuroscience, Aarhus University hospital, Aarhus University, Aarhus, Denmark; ${ }^{3}$ Cognitive Brain Research Unit, Cognitive Science, Institute of Behavioural Sciences, University of Helsinki, Helsinki, Finland; ${ }^{4}$ Department of Psychology, Lund University, Lund, Sweden; ${ }^{5}$ Department of Women's and Children's Health, Uppsala University, Uppsala, Sweden; ${ }^{6}$ Finnish Institute of Occupational Health, Helsinki, Finland; ${ }^{7}$ Children's Hospital, Helsinki University Hospital and University of Helsinki, Helsinki, Finland. Correspondence: Holger Hövel (holger.hovel@med.lu.se)

Received 13 May 2014; accepted 1 October 2014; advance online publication 25 February 2015. doi:10.1038/pr.2015.7 
5 -y-old children born with mean gestational ages below $28 \mathrm{GW}$ $(2,16)$. In children born at less than $32 \mathrm{GW}$ studied at $2 \mathrm{y}$ of age, MMN responses reflected abnormal phoneme discrimination, which was related to a slower native language acquisition (17).

In this study, we aimed to investigate if AERP recorded at preschool age in children born very preterm (VP) at less than $32 \mathrm{GW}$ are associated with neurocognitive outcome. We hypothesized that children who have abnormal results on cognitive or behavioral scales show AERP responses that differ from children who develop normally. As a secondary aim, we investigated the correlates of neonatal clinical risk factors with cortical auditory processing.

\section{RESULTS}

\section{Subjects}

Of the $87 \mathrm{VP}$ infants recruited at birth, born with a gestational age less than $32 \mathrm{wk}$, three died during the neonatal period, one could not be contacted, and one had moved abroad. The parents of 10 children declined to participate in this study. Two children consequently declined the fixation of EEG electrodes. Thus, 70 VP children born at 23.9-31.7 GW had a complete AERP recording. Perinatal characteristics of these are shown in Table 1. The children were assessed at the age of $61.4 \pm 2.4 \mathrm{mo}(58.6 \pm 2.5 \mathrm{mo}$ corrected for gestational age). All children passed the national hearing test at $4 \mathrm{y}$ of age and none used a hearing aid.

Table 1. Perinatal characteristics of all children with AERP recordings as well as of the subgroups with and without brain damage

\begin{tabular}{|c|c|c|c|}
\hline & $\begin{array}{l}\text { All children } \\
\qquad(n=70)\end{array}$ & $\begin{array}{l}\text { Brain damage } \\
\qquad(n=10)\end{array}$ & $\begin{array}{l}\text { No brain damage } \\
\qquad(n=60)\end{array}$ \\
\hline Gestational age (weeks) & $27.4 \pm 1.9(23.9-31.7)$ & $27.0 \pm 2.0(25.1-31.7)$ & $27.6 \pm 1.9(23.9-31.7)$ \\
\hline Gestational age $<28$ wk & $44(63)$ & $7(70)$ & $37(62)$ \\
\hline Birth weight (g) & $996 \pm 288(550-2025)$ & $924 \pm 231(605-1,205)$ & $1,009 \pm 299(550-2,025)$ \\
\hline Small for gestational age ( $\leq-2 \mathrm{SD})$ & $21(30)$ & $3(30)$ & $18(30)$ \\
\hline Chorioamnionitis & $2(3)$ & $1(10)$ & $1(2)$ \\
\hline Maternal pre-eclampsia & $17(24)$ & $0(0)$ & $17(28)$ \\
\hline Antenatal steroids & $69(99)$ & $10(100)$ & $59(98)$ \\
\hline Birth through cesarean section & $49(70)$ & $5(50)$ & $44(73)$ \\
\hline Male gender & $35(50)$ & $7(70)$ & $28(47)$ \\
\hline Twin & $29(41)$ & $5(50)$ & $24(40)$ \\
\hline Triplet & $5(7)$ & $0(0)$ & $5(8)$ \\
\hline Need for inotropics & $29(41)$ & $6(60)$ & $23(38)$ \\
\hline Operation for patent ductus arteriosus & $13(19)$ & $3(30)$ & $10(17)$ \\
\hline Sepsis (verified by blood culture) & $18(26)$ & $4(40)$ & $14(23)$ \\
\hline Necrotizing enterocolitis & $2(3)$ & $1(10)$ & $1(2)$ \\
\hline $\begin{array}{l}\text { Intracranial hemorrhage (grade I-III or periventricular } \\
\text { hemorrhagic infarction) }\end{array}$ & $15(21)$ & $7(70)$ & $8(13)$ \\
\hline $\begin{array}{l}\text { Severe intracranial hemorrhage (grade III or } \\
\text { periventricular hemorrhagic infarction) }\end{array}$ & $4(6)$ & $4(40)$ & $0(0)$ \\
\hline Shunt for posthemorrhagic hydrocephalus & $2(3)$ & $2(20)$ & $0(0)$ \\
\hline Periventricular leukomalacia & $8(11)$ & $8(80)$ & $0(0)$ \\
\hline Neonatal brain damage & $10(14)$ & $10(100)$ & $0(0)$ \\
\hline Days on ventilator & $6.7 \pm 8.9$ & $8.6 \pm 8.0$ & $6.2 \pm 9.0$ \\
\hline Days on nasal continuous positive airway pressure (CPAP) & $19.4 \pm 17.3$ & $24.1 \pm 17.1$ & $18.5 \pm 17.5$ \\
\hline Days at hospital & $86 \pm 24$ & $98 \pm 28$ & $84 \pm 24$ \\
\hline Laser therapy for retinopathy of prematurity & $6(9)$ & $0(0)$ & $6(10)$ \\
\hline Bronchopulmonary dysplasia (oxygen at 36 GW) & $35(50)$ & $5(50)$ & $30(50)$ \\
\hline Weight at term (g) & $2995 \pm 518$ & $3079 \pm 598$ & $2976 \pm 511$ \\
\hline Head circumference at term $(\mathrm{cm})$ & $35.2 \pm 1.4$ & $35.5 \pm 1.5$ & $35.2 \pm 1.4$ \\
\hline Head circumference at AERP $(\mathrm{cm})$ & $50.6 \pm 1.9$ & $50.6 \pm 2.8$ & $50.6 \pm 1.8$ \\
\hline
\end{tabular}

Values as numbers (\%) or Mean \pm SD (range).

AERP, auditory event-related potentials; CPAP, 


\section{Articles | Hövel et al.}

Table 2. Cognitive test results and SDQ-scores for all children, and for children with and without neonatal brain damage

\begin{tabular}{|c|c|c|c|c|c|c|}
\hline \multirow[b]{2}{*}{ Test } & \multirow[b]{2}{*}{ Score } & \multicolumn{3}{|c|}{ All children $n=70$} & \multirow{2}{*}{$\frac{\text { Brain damage } n=10}{\text { Mean } \pm \text { SD }}$} & \multirow{2}{*}{$\frac{\text { No brain damage } n=60}{\text { Mean } \pm \text { SD }}$} \\
\hline & & $\mathrm{n}$ & Mean \pm SD & Abnormal (\%) & & \\
\hline \multirow[t]{3}{*}{ WPPSI } & VIQ & 69 & $97.5 \pm 12.0$ & $9(13)$ & $92.0 \pm 12.6$ & $98.4 \pm 11.8$ \\
\hline & PSQ & 67 & $85.0 \pm 11.6$ & $30(45)$ & $74.8 \pm 15.0$ & $86.8 \pm 10.0^{* *}$ \\
\hline & FSIQ & 69 & $94.2 \pm 13.4$ & $13(19)$ & $86.8 \pm 16.4$ & $95.5 \pm 12.6$ \\
\hline \multirow{4}{*}{ NEPSY } & VAt & 67 & $208 \pm 67$ & $12(18)$ & $253 \pm 71$ & $202 \pm 64^{*}$ \\
\hline & PP & 69 & $11.8 \pm 2.3$ & $10(14)$ & $11.2 \pm 2.6$ & $11.9 \pm 2.3$ \\
\hline & NM & 69 & $10.0 \pm 4.8$ & $18(26)$ & $7.5 \pm 3.7$ & $10.4 \pm 4.8$ \\
\hline & SR & 68 & $17.3 \pm 4.3$ & $9(13)$ & $16.7 \pm 6.0$ & $17.4 \pm 4.0$ \\
\hline SDQ & Tot & 70 & $9.1 \pm 4.6$ & $3(4)$ & $12.8 \pm 5.3$ & $8.5 \pm 4.2^{* *}$ \\
\hline
\end{tabular}

Cut-off limits for abnormal test results are described in the Methods section.

FSIQ, full-scale IQ; NM, narrative memory; PIQ, performance IQ; PP, phonological processing; SDQ, strengths and difficulties questionnaire; SR, sentence repetition; Tot = total score; VIQ, verbal IQ.

$P$ values for comparison between children with and without brain damage. ${ }^{*} P<0.05$; ${ }^{* *} P<0.01$.

\section{Cognitive Test Results}

Cognitive test results are shown in Table 2. Children with neonatal brain damage (i.e., ultrasound abnormalities) had significantly lower processing speed quotients, less correct markings (VAco) and slower performance (VAt) at the visual attention subtest, as well as more difficulties according to the Strengths and Difficulties Questionnaire.

\section{AERP and Neurocognition}

Mean responses to the standard and deviant stimuli are shown in Figure 1.

As AERP components show a strong correlation with age at examination (16), all statistics including AERP data were corrected for age. Age at examination correlated significantly with the P1 latency $(r=-0.423 ; P<0.001)$, but not with any other AERP data.

Cognitive test results were related to AERP. Faster VAt correlated with shorter P1 $(r=0.249 ; P=0.048)$ and N2 latencies $(r=0.319 ; P=0.010)$. Children with abnormal VAt had longer N2 latencies $(266.0 \pm 25.1$ vs. $244.9 \pm 19.3 \mathrm{~ms} ; P=0.003)$ and a trend to longer $P 1$ latencies $(125.8 \pm 8.4$ vs. $116.1 \pm 16.7 \mathrm{~ms}$; $P=0.065)$. P1 and N2 amplitudes, however, were not related to cognitive test results.

The most consistent associations between cognitive test results and mean amplitudes in the time windows were found for the duration deviant (Table 3). From $150 \mathrm{~ms}$ after stimulus change onset, children with better cognitive test results (except for VAt) showed consistently more positive mean amplitudes in all time windows. At 300-350 ms, mean amplitudes were significantly higher for three of nine cognitive subtests for this deviant.

These associations were confirmed by comparing children with abnormal vs. normal cognitive test results in the three time windows 300-450 ms after change onset for the duration deviant (Table 4). Children with abnormal results in all nine subtests had lower mean amplitudes in these time windows

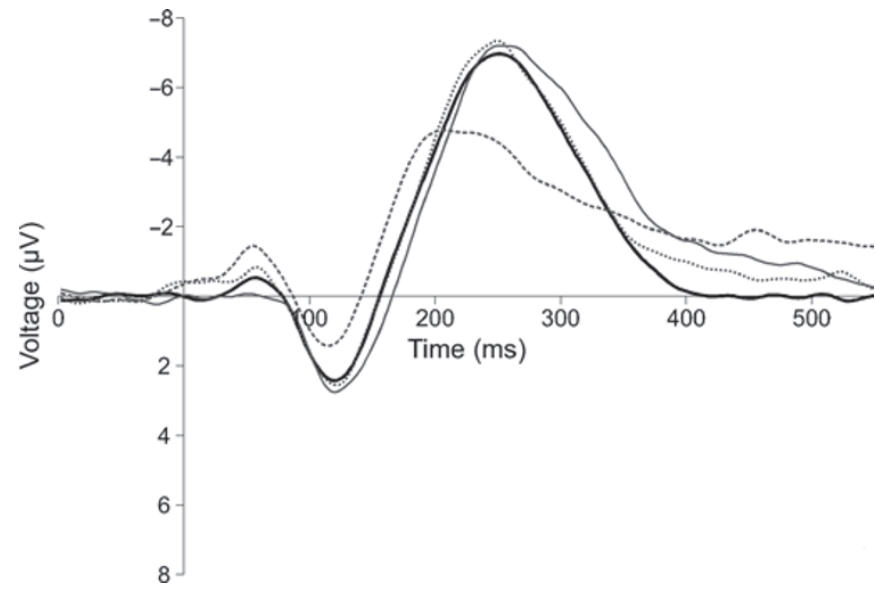

Figure 1. Grand average AERP responses to the standard stimulus and the three deviant stimuli for all $n=70$ children. The positive peak around $100 \mathrm{~ms}$ is the $\mathrm{P} 1$, the negative peak around $250 \mathrm{~ms}$ the $\mathrm{N} 2$ response. Bold black line $=$ standard. Dashed line $=$ duration deviant ; dotted line $=$ direc tion deviant; grey line = frequency deviant.

than those with normal results, except for phonological processing in the 300-400 ms time windows. These more negative mean amplitudes reached significance for the VAco subtest in all three time windows and for the VAt subtest in the 400$450 \mathrm{~ms}$ time window.

Regarding the frequency deviant, children with abnormal processing speed quotients had more negative amplitudes in all seven time windows between $150-500 \mathrm{~ms}$ than children performing normally, reaching significance in the $350-400 \mathrm{~ms}$ time window $(-2.32 \pm 1.78$ vs. $-1.25 \pm 2.34 \mu \mathrm{V}$; $P=0.011$ ). Children with abnormal VAco scores had significantly lower mean amplitudes to the direction deviant in all seven time windows, reaching significance in the 200-250 $(-1.62 \pm 2.74$ vs. $-0.02 \pm 2.27 \mu \mathrm{V} ; P=0.044)$ and $300-350 \mathrm{~ms}$ time windows $(-1.72 \pm 2.09$ vs. $0.23 \pm 2.37 \mu \mathrm{V} ; P=0.014)$. In contrast, faster VAt (better performance) correlated with 
Table 3. Correlation coefficients ( $r$ or $\beta$ when corrected for head circumference) between mean amplitudes in 50-ms time windows after stimulus change onset for the duration deviant and cognitive test results

\begin{tabular}{|c|c|c|c|c|c|c|c|c|}
\hline Test subtest & $n$ & $100-150 \mathrm{~ms}$ & $150-200 \mathrm{~ms}$ & $200-250 \mathrm{~ms}$ & $250-300 \mathrm{~ms}$ & $300-350 \mathrm{~ms}$ & $350-400 \mathrm{~ms}$ & $400-450 \mathrm{~ms}$ \\
\hline \multicolumn{9}{|l|}{ WPPSI } \\
\hline VIQ & 69 & -0.072 & 0.069 & 0.156 & 0.148 & $0.229 * * *$ & 0.138 & 0.092 \\
\hline PSQ & 67 & -0.055 & 0.157 & 0.151 & 0.191 & $0.355^{* *}$ & $0.354^{* *}$ & $0.274^{*}$ \\
\hline FSIQ & 69 & -0.112 & 0.071 & 0.152 & 0.108 & $0.255^{*}$ & 0.197 & 0.145 \\
\hline VAco & 64 & -0.138 & 0.151 & 0.067 & 0.022 & $0.221^{* * *}$ & $0.340^{* *}$ & $0.235^{* * *}$ \\
\hline Vat & 64 & 0.079 & 0.192 & -0.030 & 0.025 & 0.006 & -0.067 & $-0.254^{* * *}$ \\
\hline PP & 65 & 0.109 & $0.267^{*}$ & $0.255^{*}$ & 0.166 & $0.207^{* * *}$ & 0.099 & 0.106 \\
\hline NM & 65 & -0.121 & 0.166 & 0.151 & 0.109 & 0.193 & 0.150 & 0.116 \\
\hline$S R$ & 65 & 0.008 & 0.140 & 0.116 & 0.070 & $0.252^{*}$ & 0.132 & 0.175 \\
\hline
\end{tabular}

The time windows 250-300 ms after stimulus change onset is the window of the MMN response only, the window $400-450$ ms is the window of the P3a response only. The time windows between $300-400$ ms are overlapping time windows of the MMN and P3a reponses.

${ }^{*} P<0.05 ;{ }^{* *} P<0.01 ;{ }^{* * *} P=0.05-0.1$.

FSIQ, full-scale IQ; MMN, mismatch negativity; NM, narrative memory; PIQ, performance IQ; PP, phonological processing; SR, sentence repetition; $V I Q$, verbal IQ.

lower mean amplitudes to this deviant in all seven time windows (significant for $150-200 \mathrm{~ms}, r=0.249 ; P=0.042$ and 250-300 ms, $r=0.291 ; P=0.017)$.

\section{AERP After Neonatal Brain Damage}

Perinatal data for all children and children with and without brain damage are shown in Table 1. Mean amplitudes in time windows for children with and without neonatal brain damage are shown in Table 5. Average difference curves to the three deviants for both groups are shown in Figure 2.

Based on the grand average curves, we defined the MMN time windows for the frequency deviant as $200-350 \mathrm{~ms}$, for the direction deviant as $150-300 \mathrm{~ms}$, and for the duration deviant as $250-400 \mathrm{~ms}$ after stimulus change onset. The P3a time windows defined accordingly were $300-450 \mathrm{~ms}$ after stimulus change onset for all three deviants.

In the seven time windows between $100-450 \mathrm{~ms}$, mean amplitudes of all three deviants were more negative in children with neonatal brain injury (i.e., stronger response in the MMN and weaker response in the P3a time windows), except for the $400-450 \mathrm{~ms}$ P3a time window for the frequency deviant. This group difference was significant in the $100-150 \mathrm{~ms}$ time window of the direction deviant and in the four time windows between 150-350 ms for the duration deviant.

No group differences in latencies and amplitudes of the P1 and $\mathrm{N} 2$ responses were found.

\section{AERP Time Windows and Neonatal Morbidity}

Correlations between mean amplitudes in time windows and neonatal morbidity are shown in Supplementary Table S1 online.

Neonatal morbidity was associated with AERP changes. With decreasing GW, more negative mean amplitudes were found at $400-450 \mathrm{~ms}$ for the direction deviant and at $200-250 \mathrm{~ms}$ for the duration deviant.
Sepsis $(n=18)$ was associated with longer latencies of P1 ( $\beta=0.345 ; P=0.020)$ as well as with less negative mean amplitudes in early time windows of the direction $(200-250 \mathrm{~ms})$ and duration deviants (100-150 ms). Lower 5-min Apgar scores predicted more negative mean amplitudes in early time windows to the frequency $(100-150 \mathrm{~ms})$ and direction deviants $(150-200 \mathrm{~ms})$. Retinopathy of prematurity requiring laser therapy for $(n=6)$ predicted lower $\mathrm{N} 2$ amplitudes $(\beta=-0.336$; $P=0.046)$.

While sepsis and BPD $(n=35)$ correlated with less negative mean amplitudes in early time windows for the duration deviant (100-150 ms), longer mechanical ventilation strongly correlated with more negative mean amplitudes for this deviant (100-300 ms).

\section{DISCUSSION}

Our study shows that shorter latencies of the P1 and N2 components and more positive mean amplitudes in the MMN and P3a time windows were associated with better cognitive test results. As MMN by definition is a negative and P3a a positive deflection, more positive mean amplitudes in MMN and P3a time windows equal to weaker MMN and stronger P3a responses. Conversely, immaturity, most neonatal risk factors, and especially neonatal cerebral ultrasound abnormalities were associated with a negative displacement of the difference waveforms, generating an increase in $\mathrm{MMN}$ and a decrement in P3a amplitude.

Early AERP time windows reflect early cortical processes such as encoding and early processing while later time windows represent more advanced cognitive processes like change detection, auditory discrimination, and attentional switch $(4,9,11)$. In our study, amplitudes in early time windows were influenced by gestational age, neonatal brain damage, sepsis, 5-min Apgar scores, days on a ventilator, and BPD. Conversely, amplitudes in later time windows were influenced 


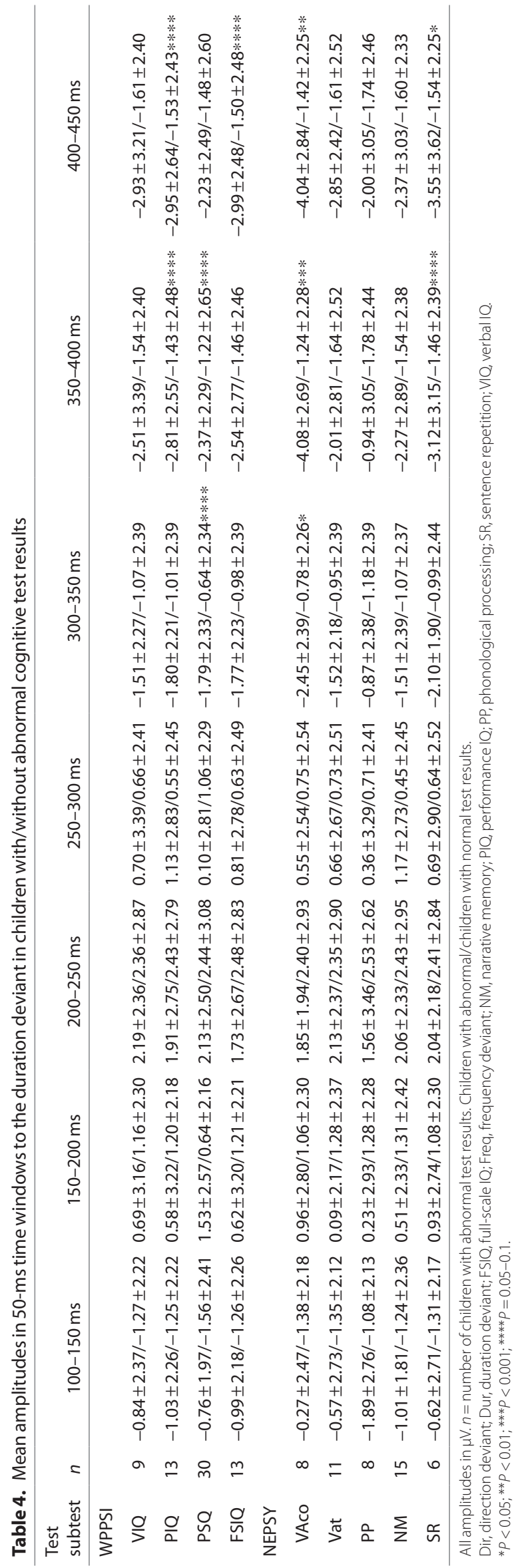

by gestational age, neonatal brain damage, and 5-min Apgar scores only. Thus, different neonatal risk factors seem to influence the development of central auditory processing differently.

While neonatal morbidity mostly affected time windows before $250 \mathrm{~ms}$, neuropsychological test performance was most strongly associated with later AERPs after $300 \mathrm{~ms}$. This is reasonable as more advanced cortical auditory processing correlated with cognition (such as the MMN and P3a components) appears in later AERP time windows. Brain damage had an impact on all examined time points including the earliest time windows reflecting auditory encoding before the MMN. Thus, brain damage is influencing the whole spectrum of cortical sound encoding and processing. Interestingly, time windows affected by brain damage are identical with the time windows associated with neonatal morbidity (causing brain damage) and cognitive outcome (highly influenced by brain damage). As no detailed cerebral imaging was performed after the neonatal ultrasound scans in all children, it remains unclear whether other injuries than intraventricular hemorrhage and periventricular cysts might affect AERP.

Lower P1 amplitudes in children born VP have been described earlier $(2,16)$. In this study, the P1 amplitude tended to increase with increasing gestational age, but it was not influenced by brain damage or other morbidity. Thus, environmental factors common to all VP infants rather than prematurity-related morbidity seem to determine the development of auditory encoding. Decreasing P1 with increasing prematurity and brain damage might reflect increased habituation to repetitive stimuli in these children (18). As the AERPs of the present study were recorded at preschool age, our results suggest that early extrauterine auditory environment in the neonatal period may modify the auditory network even in a longer perspective. The auditory environment is different between VP and term-born infants during the last trimester. Both favorable intrauterine influences and unfavorable effects of the NICU environment such as noisy ventilator support devices and disturbing alarms $(19,20)$ may have an impact on later auditory processing.

In addition to stimulus characteristics and magnitude of stimulus change, component latencies in children are influenced by continued myelination, synaptic differentiation, and specialization $(21,22)$. Advanced MRI studies have shown delayed microanatomical differentiation and myelination in white matter tracts in preterm infants (23). More mature auditory pathways in the brainstem and pons have been associated with shorter latencies on brainstem auditory-evoked potentials $(24,25)$. While auditory connectivity is difficult to assess due to the small size and vicinity to adjacent structures of the relevant tracts, advanced volumetric brain studies in preterm children have revealed reduced volumes in temporal brain regions (26). Thus, it may be that both delayed myelination and impaired cortical differentiation may explain the increased AERP latencies in children with lower gestational age, sepsis, and lower 5-min Apgar scores.

Furthermore, our study shows significant correlations between AERP measures and cognitive outcome at preschool 
Table 5. Mean amplitudes in $\mu \mathrm{V}$ (mean $\pm \mathrm{SD}$ ) of the deviant-minus-standard difference waves in the 50-ms time windows between $100-450 \mathrm{~ms}$ from stimulus change

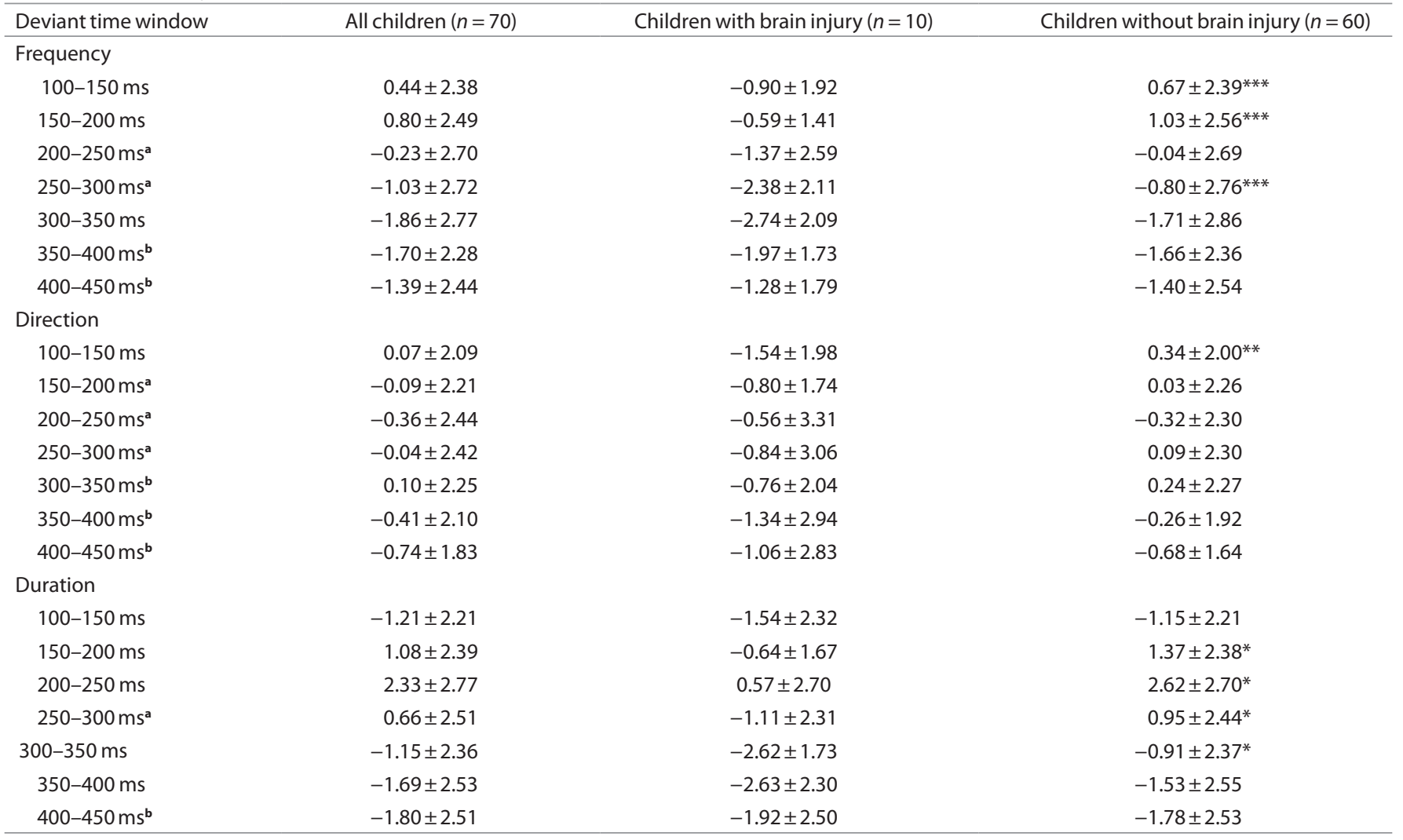

aTime windows when only mismatch negativity appeared. ${ }^{\circ}$ Time windows when only P3a appeared.

${ }^{*} P<0.05 ;{ }^{* *} P<0.01 ;{ }^{* *} P=0.05-0.1$ for comparison between children with and without brain damage (adjusted for head circumference when appropriate).

a

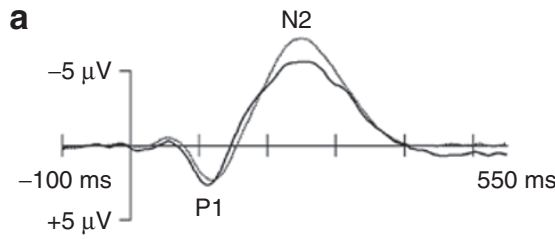

C

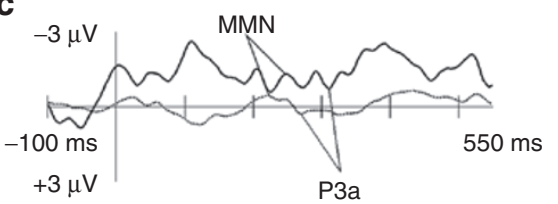

b

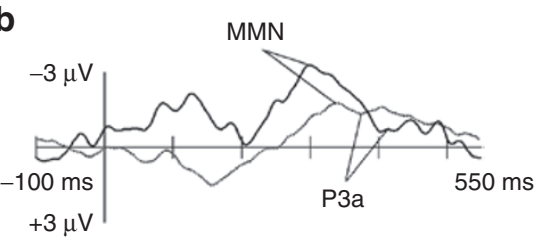

d

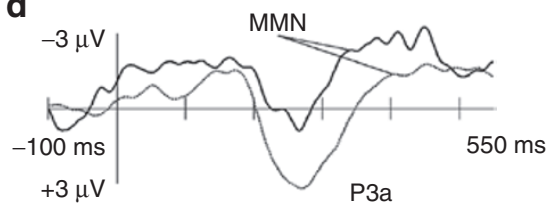

Figure 2. Comparison between children with and without neonatal brain damage. Average auditory event-related potentials standard curve (a) and deviant-minus-standard difference curves to the frequency (b), direction (c), and duration deviants (d) of children with (full line) and without (dotted line) brain damage. The main responses are marked on each curve. No P3a can be observed on the average curve of the duration. Stimulus onset and change onset is at $0 \mathrm{~ms}$ with the exception of change onset for the duration deviant $(50 \mathrm{~ms})$.

age. Very few earlier studies have addressed these correlations in preterm infants. One study has failed to show such correlations at $2 \mathrm{y}$ of age (27), while a recent study using speech sounds on term-born and premature newborns showed that AERP at term was predictive of outcome in infancy (28).

Alterations in AERP and particularly MMN have been shown in children with learning disabilities (29), reading and language-related disorders (17), autism spectrum disorders
$(6,13,30)$, distractibility, and attention deficit hyperactivity disorder (30). In our study, AERP difference waves to all three deviants in children with brain damage and lower cognitive scores were displaced negatively, leading to stronger MMN and weaker P3a responses. This is in accordance with a study showing more negative responses to a frequency deviant during infancy in full-term children with neonatal brain damage (27). Perinatal brain damage together with plasticity during 
subsequent brain development may considerably alter different phases of auditory processing (31). As ERP amplitudes and polarities depend on the magnitude of synaptic activation, the localization and orientation of the cortical or subcortical generators, excitatory or inhibitory nature of the input, and reception via synapses distal or proximal to the cell bodies (32), the negative AERP displacement is an additional indicator of altered brain maturation after neonatal brain damage and degree of immaturity at birth.

Investigation of AERP as a prognostic tool has only been evaluated recently and in small patient groups. This is so far the largest AERP study on preterm children, and the only study investigating the impact of prenatal and neonatal clinical factors on AERP responses beyond infancy. Strengths of our study are first that the study infants were consecutively recruited after birth, and more than $80 \%$ participated in the follow-up study at school age. Second, the success rate of the recordings was very high, with only two children (3\%) not cooperating sufficiently for reliable recordings. In previous studies, up to $20 \%$ of recordings were rejected due to technical problems (e.g., refs $(2,12)$ ).

A caveat has been the use of multiple comparisons. A Bonferroni correction would have made most of the significant results disappear because of small group sizes and small differences between groups. Thus, in order to avoid reporting accidental correlations, we only present results that are consistent (or supported by adjacent-albeit only borderline significant_changes) between cognitive tests, adjacent time windows, or between at least two of the deviants. However, relevant findings may be excluded with this approach.

Unfortunately, our AERP paradigm was not designed to detect specific adverse neuropsychological outcomes. Other paradigms may increase the prognostic value for a specific impairment. However, AERP recordings and analyses are time consuming, and in spite of modern paradigms $(7,33)$, the number of deviants testable is limited. Our aim was to apply an easy paradigm testing several important aspects of sound processing such as frequency, duration, and spatial perception. However, this paradigm does not seem to be sufficiently specific to be used as a prognostic tool on an individual basis.

We conclude that AERP measures at preschool age are influenced by gestational age at birth, neonatal brain damage and morbidity, and are associated with cognitive outcome at preschool age.

\section{METHODS \\ Study Population}

Preterm infants with a gestational age below $32 \mathrm{wk}$, born at Lund University Hospital between September 2000 and February 2003 and treated at the NICU were recruited into a prospective cohort study. Major congenital malformations were the only exclusion criterion. A total of 87 infants were enrolled.

Clinical data were collected prospectively during the neonatal period. Neonatal cerebral ultrasound scans were performed routinely at 1,3 , and $7 \mathrm{~d}, 3 \mathrm{wk}$, and at term. As severe intracranial hemorrhage, we defined intraventricular hemorrhage with ventricular dilation (grade III) and/or periventricular hemorrhagic infarction. As neonatal brain damage, we defined severe intracranial hemorrhage and/or periventricular cysts on ultrasound at any time point up to term age.

The age at AERP examination was calculated from the time corresponding to term age. Informed, written parental consent was obtained both at recruitment and before the AERP recording at 4-5.5 y of age. The Regional Ethics Review Board, Lund, Sweden approved the study protocol prior to the start of the study.

\section{AERP Recording and Stimuli}

Auditory stimuli were delivered binaurally through headsets at $60 \mathrm{~dB}$ sound pressure level to the children while they watched a silenced movie in a sound-attenuated room. $\mathrm{Ag} / \mathrm{Ag}-\mathrm{Cl}$ electrodes were attached at electrode sites F3, Fz, F4, C3, Cz, C4, P3, Pz, P4, T3, and T4 according to the International 10-20 System, as well as four electro-oculogram electrodes. The EEG was referenced to the average of mastoid electrodes. An EEG (bandpass $0.1-70 \mathrm{~Hz}$, sampling rate 500 $\mathrm{Hz}$ ) was recorded using the NeuroScan 4.3 system (Compumedics, Abbotsford, Australia).

The sound stimuli were presented as an oddball paradigm consisting of a standard tone (probability of 70\%), randomly replaced by one of three infrequent deviant tones (10\% probability for each). The time interval between the start of successive stimuli was $533 \mathrm{~ms}$. The standard tone was a sinusoidal $1000 \mathrm{~Hz}$ tone with a duration of $100 \mathrm{~ms}$. The frequency deviant differed from the standard by a $10 \%$ higher pitch $(1100 \mathrm{~Hz})$. An apparent direction deviant differed in perceived sound source location, achieved by a sound onset difference between the left and the right side of $750 \mu$ s. The third deviant differed in duration, lasting only $50 \mathrm{~ms}$. Otherwise, all deviants were identical to the standard tone.

Stimuli were presented in three blocks of 610 sounds, each block containing all deviant types. Each deviant was followed by at least one standard tone. In total, the standard tone was presented 1,290 times, while each deviant type was presented 180 times. The duration of the experiment was about $15 \mathrm{~min}$.

\section{AERP Averaging and Analysis}

AERP data analysis was performed using a NeuroScan 4.5 system (Compumedics). The continuous EEG was filtered offline (bandpass $0.5-30 \mathrm{~Hz}$ ). The data were divided into 650 - $\mathrm{ms}$ epochs $(100 \mathrm{~ms}$ before to $550 \mathrm{~ms}$ after stimulus onset). The epochs were baseline corrected to the prestimulus interval. Epochs with amplitudes exceeding $\pm 100 \mu \mathrm{V}$ on any electrode were rejected, as well as all epochs following a deviant stimulus epoch. The epochs for the standard stimulus and those for the three deviant stimuli were averaged into separate averages for each subject and electrode. The final data set consisted of, on average, 101 accepted trials per deviant type (range: 41-162).

The AERP curves for standard and deviant stimuli in all subjects were averaged into grand average mean AERP curves. An averaged curve from the six frontal and central electrodes was used to define the individual latencies of the main peaks for each participant.

The P1 peak was defined as the most positive peak in the interval $130 \pm 50 \mathrm{~ms}$, and the $\mathrm{N} 2$ peak as the most negative peak in the interval $250 \pm 80 \mathrm{~ms}$.

Difference waveforms were obtained by subtracting the response to the standard from that to the deviant stimulus, individually for each child, using the averaged curves from the six frontal and central electrodes. In order to reduce the influence of noise, combined mean amplitudes for these six electrodes in successive $50 \mathrm{~ms}$ time windows from 100 to $450 \mathrm{~ms}$ after stimulus change onset were calculated for each subject. As MMN time window for each deviant, we defined the $50 \mathrm{~ms}$ time window with the MMN on the grand average mean curve and the previous and following $50 \mathrm{~ms}$ time windows (in total, three successive time windows). The P3a time windows were defined accordingly.

\section{Neuropsychological Tests}

On the same day as the AERP recordings, the children were tested using all subtests of the Wechsler Preschool and Primary Scale of intelligence-revised (WPPSI-R) (34) and the subtests Visual attention, Phonological Processing, Narrative memory and Sentence repetition of A Developmental Neuropsychological Assessment (NEPSY) 
(35). The tests were performed by one of two psychologists trained in these test methods and blinded to both neonatal morbidity and AERP results.

The parents filled in the SDQ (36), in which we analyzed the total score. Length, body weight, and head circumference were registered.

The age range of the children at assessment was 4.3-5.3 y. In order to avoid effects of different test protocols in NEPSY for children below and above $5 \mathrm{y}$ of age, all children were tested using the protocols for the age group below $5 \mathrm{y}$. Where no percentiles for 5 -y olds were available for these protocols, percentiles for 4-y olds were used even for the children 5 to $5.3 \mathrm{mo}$ of age. In order to elucidate different aspects of the visual attention subtest of NEPSY, we analyzed the number of correct markings (VAco) and the time to complete this test (VAt) separately.

Children scoring below 85 in WPPSI-R, on the 25th percentile or below on a NEPSY subtest and higher than 16 on the Strengths and Difficulties Questionnaire were considered abnormal for this subtest or score.

To exclude the effect of severe cognitive impairment on NEPSY test results, children with full-scale IQ $\leq 70$ in WPPSI-R $(n=4)$ were not included in the NEPSY analysis.

\section{Statistical Analyses}

Statistical analyses were performed with PASW Statistics 18 for Windows software (IBM SPSS Statistics, Chicago, IL).

As we in an earlier study had found a significant association between age at examination and AERP latencies (16), and as head size may have an impact on AERP measures, all AERP measures correlating with these factors $(P<0.1$ on Pearson bivariate correlations) were corrected for respective factor. Thus, we corrected for head circumference in the time windows between 150-250 and 350-400 ms for the frequency deviant, between $350-400 \mathrm{~ms}$ for the direction deviant, and between $200-250$ and $350-450 \mathrm{~ms}$ for the duration deviant.

AERP measures and cognitive test results in children with and without neonatal brain damage were compared using $t$-test. Where correction for age at examination or head circumference was necessary, we used binary logistic regression analysis.

Associations between AERP measures and cognitive test results/ Strengths and Difficulties Questionnaire score were tested using bivariate correlations (Pearson). Where correction for age at examination or head circumference was necessary, we used linear or binary logistic regression analysis. Children with abnormal and normal test results/scores were compared using $t$-test.

The influence of neonatal clinical data on AERP measures were tested using multiple regression analysis, with AERP measures as dependent and neonatal clinical data as independent variables. In the primary model we included the prenatal factors sex, GA (continuous variable), and small for GA (SGA, defined as birth weight below -2 $\mathrm{SD}$ for gestation). In the secondary model, we further added the neonatal clinical variables 5-min Apgar, days on ventilator, sepsis, laser therapy for retinopathy of prematurity, and BPD (defined by need for additional oxygen at $36 \mathrm{GW}$ ), all known to have an impact on cognitive outcome. Correction for age at examination or head circumference was added where appropriate.

In order to avoid reporting accidental associations due to multiple comparisons, we only present results that are consistent between cognitive tests, adjacent time windows, or between at least two of the deviants.

A $P$ value $<0.05$ was considered significant.

\section{SUPPLEMENTARY MATERIAL}

Supplementary material is linked to the online version of the paper at http://www.nature.com/pr

\section{ACKNOWLEDGMENTS}

We thank Ann-Cathrine Berg, Anette Carnemalm, Eva Hammarstrand, Christina Helgason, and Bodil Persson for excellent help in accomplishing the testings.

\section{STATEMENT OF FINANCIAL SUPPORT}

The study was supported by grants from the Swedish Medical Research Foundation, the Swedish Research Council (grant no. 349-2007-8695), the Skåne Council Foundation for Research and Development (Lund, Sweden), the Finnish Institute of Occupational Health (Helsinki, Finland), Academy of Finland (grant nos. 135161 and 135304, Helsinki, Finland), Lund University funds (Lund, Sweden), and from the Allmänna BB, Samariten, and Nils Svenningsen Prematurity Foundations (all Sweden).

Disclosure: Nothing to disclose.

\section{REFERENCES}

1. Aarnoudse-Moens CS, Weisglas-Kuperus N, van Goudoever JB, Oosterlaan J. Meta-analysis of neurobehavioral outcomes in very preterm and/or very low birth weight children. Pediatrics 2009;124:717-28.

2. Mikkola K, Kushnerenko E, Partanen E, et al. Auditory event-related potentials and cognitive function of preterm children at five years of age. Clin Neurophysiol 2007;118:1494-502.

3. Kral A, Eggermont JJ. What's to lose and what's to learn: development under auditory deprivation, cochlear implants and limits of cortical plasticity. Brain Res Rev 2007;56:259-69.

4. Cunningham J, Nicol T, Zecker S, Kraus N. Speech-evoked neurophysiologic responses in children with learning problems: development and behavioral correlates of perception. Ear Hear 2000;21:554-68.

5. Wunderlich JL, Cone-Wesson BK. Maturation of CAEP in infants and children: a review. Hear Res 2006;212:212-23.

6. Lepistö T, Kujala T, Vanhala R, Alku P, Huotilainen M, Näätänen R. The discrimination of and orienting to speech and non-speech sounds in children with autism. Brain Res 2005;1066:147-57.

7. Lovio R, Näätänen R, Kujala T. Abnormal pattern of cortical speech feature discrimination in 6-year-old children at risk for dyslexia. Brain Res 2010;1335:53-62.

8. Benasich AA, Choudhury N, Friedman JT, Realpe-Bonilla T, Chojnowska C, Gou Z. The infant as a prelinguistic model for language learning impairments: predicting from event-related potentials to behavior. Neuropsychologia 2006;44:396-411.

9. Näätänen R, Tervaniemi M, Sussman E, Paavilainen P, Winkler I. "Primitive intelligence" in the auditory cortex. Trends Neurosci 2001;24:283-8.

10. Morr ML, Shafer VL, Kreuzer JA, Kurtzberg D. Maturation of mismatch negativity in typically developing infants and preschool children. Ear Hear 2002;23:118-36.

11. Alho K, Winkler I, Escera C, et al. Processing of novel sounds and frequency changes in the human auditory cortex: magnetoencephalographic recordings. Psychophysiology 1998;35:211-24.

12. Huttunen-Scott T, Kaartinen J, Tolvanen A, Lyytinen H. Mismatch negativity (MMN) elicited by duration deviations in children with reading disorder, attention deficit or both. Int J Psychophysiol 2008;69:69-77.

13. Lepistö $T$, Nieminen-von Wendt $T$, von Wendt $L$, Näätänen R, Kujala T. Auditory cortical change detection in adults with Asperger syndrome. Neurosci Lett 2007;414:136-40.

14. Fellman V, Hellström-Westas L, Norman M, et al. One-year survival of extremely preterm infants after active perinatal care in Sweden. JAMA 2009;301:2225-33.

15. Fellman V, Kushnerenko E, Mikkola K, Ceponiene R, Leipala J, Naatanen R. Atypical auditory event-related potentials in preterm infants during the first year of life: a possible sign of cognitive dysfunction? Pediatr Res 2004;56:291-7.

16. Hövel H, Partanen E, Huotilainen M, Lindgren M, Rosén I, Fellman V. Auditory event-related potentials at preschool age in children born very preterm. Clin Neurophysiol 2014;125:449-56.

17. Jansson-Verkasalo E, Ruusuvirta T, Huotilainen M, et al. Atypical perceptual narrowing in prematurely born infants is associated with compromised language acquisition at 2 years of age. BMC Neurosci 2010;11:88.

18. Sambeth A, Maes JH, Quian Quiroga R, Coenen AM. Effects of stimulus repetitions on the event-related potential of humans and rats. Int J Psychophysiol 2004;53:197-205.

19. Gonzalez-Gomez N, Nazzi T. Phonotactic acquisition in healthy preterm infants. Dev Sci 2012;15:885-94.

20. Wachman EM, Lahav A. The effects of noise on preterm infants in the NICU. Arch Dis Child Fetal Neonatal Ed 2011;96:F305-9.

21. Ponton CW, Eggermont JJ, Kwong B, Don M. Maturation of human central auditory system activity: evidence from multi-channel evoked potentials. Clin Neurophysiol 2000;111:220-36. 


\section{Articles Hövel et al.}

22. Wunderlich JL, Cone-Wesson BK. Effects of stimulus frequency and complexity on the mismatch negativity and other components of the cortical auditory-evoked potential. J Acoust Soc Am 2001;109:1526-37.

23. Thompson DK, Lee KJ, Egan GF, et al. Regional white matter microstructure in very preterm infants: predictors and 7 year outcomes. Cortex 2014;52:60-74.

24. Kaukola T, Perhomaa M, Vainionpaa L, et al. Apparent diffusion coefficient on magnetic resonance imaging in pons and in corona radiata and relation with the neurophysiologic measurement and the outcome in very preterm infants. Neonatology 2010;97:15-21.

25. Reiman M, Parkkola R, Johansson R, et al.; PIPARI Study Group. Diffusion tensor imaging of the inferior colliculus and brainstem auditory-evoked potentials in preterm infants. Pediatr Radiol 2009;39:804-9.

26. Gousias IS, Edwards AD, Rutherford MA, et al. Magnetic resonance imaging of the newborn brain: manual segmentation of labelled atlases in termborn and preterm infants. Neuroimage 2012;62:1499-509.

27. Leipälä JA, Partanen E, Kushnerenko E, Huotilainen M, Fellman V. Perinatal cerebral insults alter auditory event-related potentials. Early Hum Dev 2011;87:89-95.

28. Maitre NL, Lambert WE, Aschner JL, Key AP. Cortical speech sound differentiation in the neonatal intensive care unit predicts cognitive and language development in the first 2 years of life. Dev Med Child Neurol 2013;55:834-9.
29. Kraus N, McGee TJ, Carrell TD, Zecker SG, Nicol TG, Koch DB. Auditory neurophysiologic responses and discrimination deficits in children with learning problems. Science 1996;273:971-3.

30. Gumenyuk V, Korzyukov O, Escera C, et al. Electrophysiological evidence of enhanced distractibility in ADHD children. Neurosci Lett 2005;374:212-7.

31. Johnston MV. Plasticity in the developing brain: implications for rehabilitation. Dev Disabil Res Rev 2009;15:94-101.

32. Wood CC. Generators of event-related potentials. In: Halliday AM, Butler SR, Paul R (eds). A Textbook of Clinical Neurophysiology. New York: Wiley, 1987:535-67.

33. Partanen E, Vainio M, Kujala T, Huotilainen M. Linguistic multifeature MMN paradigm for extensive recording of auditory discrimination profiles. Psychophysiology 2011;48:1372-80.

34. Wechsler D. Manual for the Wechsler Preschool and Primary Scale of Intelligence-Revised. San Antonio, TX: Psychological Corp, 1989.

35. Korkman M, Kirk U, Kemp SL. NEPSY - a developmental neuropsychological assessment. San Antonio, TX: Psychological Corporation, 1998.

36. Goodman R. The Strengths and Difficulties Questionnaire: a research note. J Child Psychol Psychiatry 1997;38:581-6. 